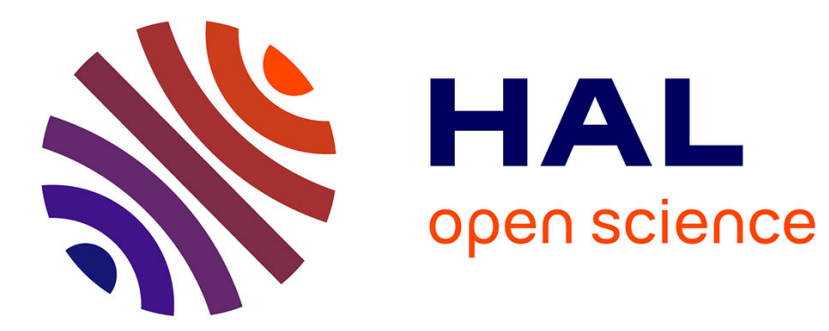

\title{
Anderson localization of electron states in a quasicrystal
}

Shuvam Sarkar, Marian Krajčí, Pampa Sadhukhan, Vipin Kumar Singh, Andrei Gloskovskii, Prabhat Mandal, Vincent Fournée, Marie-Cecile de Weerd, Julian Ledieu, Ian R Fisher, et al.

\section{- To cite this version:}

Shuvam Sarkar, Marian Krajčí, Pampa Sadhukhan, Vipin Kumar Singh, Andrei Gloskovskii, et al. Anderson localization of electron states in a quasicrystal. Physical Review B, 2021, 103 (24), 10.1103/PhysRevB.103.L241106 . hal-03403516

\section{HAL Id: hal-03403516 https://hal.science/hal-03403516}

Submitted on 26 Oct 2021

HAL is a multi-disciplinary open access archive for the deposit and dissemination of scientific research documents, whether they are published or not. The documents may come from teaching and research institutions in France or abroad, or from public or private research centers.
L'archive ouverte pluridisciplinaire HAL, est destinée au dépôt et à la diffusion de documents scientifiques de niveau recherche, publiés ou non, émanant des établissements d'enseignement et de recherche français ou étrangers, des laboratoires publics ou privés. 


\title{
Anderson localization of electron states in a quasicrystal
}

\author{
Shuvam Sarkar $\odot,{ }^{1}$ Marian Krajčí $\odot,{ }^{2}$ Pampa Sadhukhan, ${ }^{1}$ Vipin Kumar Singh, ${ }^{1}$ Andrei Gloskovskii, ${ }^{3}$ Prabhat Mandal, ${ }^{4}$ \\ Vincent Fournée, ${ }^{5}$ Marie-Cecile de Weerd, ${ }^{5}$ Julian Ledieu $\odot,{ }^{5}$ Ian R. Fisher $\odot,{ }^{6}$ and Sudipta Roy Barman $\odot^{1}$ \\ ${ }^{1}$ UGC-DAE Consortium for Scientific Research, Khandwa Road, Indore 452001, Madhya Pradesh, India \\ ${ }^{2}$ Institute of Physics, Slovak Academy of Sciences, Dúbravská cesta 9, SK-84511 Bratislava, Slovak Republic \\ ${ }^{3}$ DESY, Notkestrasse 85, D-22607 Hamburg, Germany \\ ${ }^{4}$ Saha Institute of Nuclear Physics, HBNI, 1/AF Bidhannagar, Calcutta 700 064, India \\ ${ }^{5}$ Institut Jean Lamour, UMR 7198, CNRS-Université de Lorraine, Campus ARTEM, 2 allée André Guinier, \\ Boîte Postale 50840, F-54011 Nancy, France \\ ${ }^{6}$ Geballe Laboratory for Advanced Materials and Department of Applied Physics, Stanford University, \\ Stanford, California 94305-4045, USA
}

(Received 13 November 2020; revised 27 April 2021; accepted 19 May 2021; published 9 June 2021)

\begin{abstract}
The influence of disorder on the critical electron states in a quasiperiodic lattice is a subject of intense research. In this work, we report the occurrence of Anderson localization in an icosahedral (i) polygrain quasicrystal Al-Pd-Re due to site disorder using hard x-ray photoemission spectroscopy, resistivity, and density functional theory (DFT). Photoemission spectroscopy shows that the density of states is enhanced at the Fermi level in polygrain $i$-Al-Pd-Re compared to single-grain $i$-Al-Pd-Re. In contrast, the conductivity of the former is an order of magnitude reduced compared to the latter, indicating that these electron states are localized. DFT shows that these states originate primarily from $\mathrm{Re} 5 d$-Pd $4 d$ hybridization and are enhanced in polygrain $i$-Al-Pd-Re due to compositional difference, but are broadened because of disorder that brings about Anderson localization. This is established by the Mott variable range hopping behavior of conductivity, and the estimated localization length is $23 \AA$.
\end{abstract}

DOI: 10.1103/PhysRevB.103.L241106

Anderson localization (AL) of electron states due to disorder is a fundamental concept in condensed-matter physics [1]. For randomly distributed site energies, AL occurs in the band tails, and the envelope of the electron wave function exhibits an exponential decay in real space resulting in a metal to insulator transition [2]. AL has been observed in different disordered electronic systems, such as metals with site disorder [3,4], irradiated carbon nanotubes [5], ultrathin films of topological insulators [6], and doped semiconductors [7-9], to mention a few, but has not been reported in any quasicrystal to date.

Quasicrystals discovered by Shechtman and coworkers [10] represent a unique class of material that falls in between normal crystals and amorphous systems. Unlike crystals, quasicrystals do not exhibit any translational symmetry but have an aperiodic long-range order with forbidden rotational symmetries and thus, unlike amorphous systems, are ordered. Quasicrystallinity has been reported in diverse systems; a few examples are binary and ternary intermetallic alloys [11-13], elemental metal films $[14,15]$, the self-assembled nanoparticle superlattice [16], perovskite oxide adlayers [17], and large molecular assemblies [18]. Recently reported nontrivial topological edge states in quasiperiodic systems [19,20] and a topological phase in a chalcogenide quasicrystal [21] have generated considerable excitement in this area.

The unique arrangement of atoms in quasicrystals leads to the formation of critical electron states with partially localized wave functions; however, the role of disorder in these wave functions has hardly been probed. A few theoretical studies based on the tight-binding model indeed predicted the occurrence of AL in quasicrystals with site disorder [22,23], but there has been no experimental evidence to date. Among the different quasicrystals discovered so far, the electronic properties of $i$-Al-Pd-Re, containing the $5 d$ element $\mathrm{Re}$, are the most controversial and least understood. Monocrystalline single-grain (sg) $i$-Al-Pd-Re exhibits a negative temperature coefficient of resistivity (TCR) with resistivity $\rho$ of $3-6 \mathrm{~m} \Omega \mathrm{cm}$ at $2 \mathrm{~K}[24,25]$. This behavior of $\rho(T)$ was explained by electron-electron interaction and weak localization [25-28]. In contrast, polygrain (pg) $i$-Al-Pd-Re exhibits a very pronounced negative TCR, and $\rho$ as large as $300-1000 \mathrm{~m} \Omega \mathrm{cm}$ at $4 \mathrm{~K}$ has been reported [29]. Some of the explanations suggested in the literature for such anomalously large $\rho$ in pg $i$-Al-Pd-Re include EfrosShklovskii (ES) variable range hopping (VRH) [30], doped semiconductivity [31], and a compositional difference that shifts the Fermi level $E_{F}$ in a rigid-band picture [32,33], but the possibility of AL was consistently discarded [31-34]. In spite of the large body of literature, albeit with a plethora of explanations [30,33-37], the discussions continue to date, as disagreements remain unresolved, portraying the fascinating complexity of $i$-Al-Pd-Re. Photoemission spectroscopy performed so far for $i$-Al-Pd-Re using low photon energies $(h v)$ was possibly shrouded by surface effects [38,39], such that instead of the expected pseudogap for a quasicrystal [40,41], a metallic Fermi edge was observed [42]. 

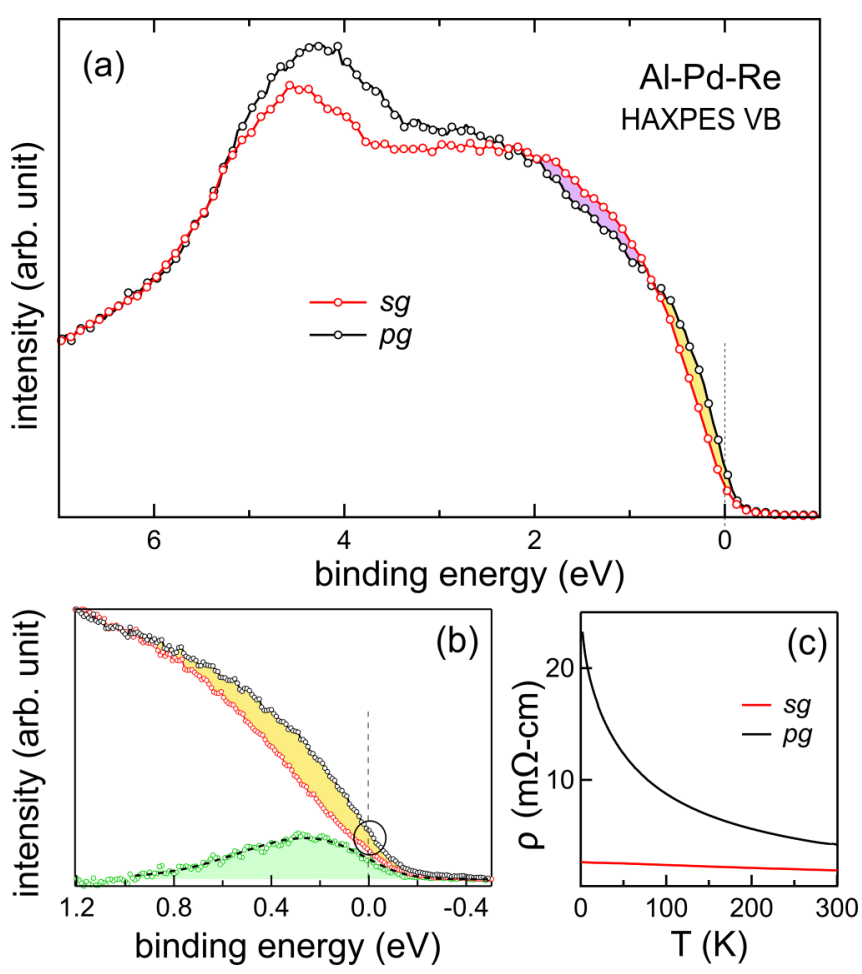

FIG. 1. (a) HAXPES valence band (VB) spectra of sg and pg $i$-Al-Pd-Re taken with $h \nu=6 \mathrm{keV}$ at $50 \mathrm{~K}$. The spectra have been normalized in the background region around $7 \mathrm{eV}$ binding energy, and the error at each data point is smaller than its size. (b) The near- $E_{F}$ region; the extra states in pg are shaded yellow. The difference spectrum (green circles and shading) is fitted with a Gaussian function (dashed black line). (c) Resistivity as a function of temperature $\rho(T)$ for pg $i$-Al-Pd-Re compared with that of sg taken from Ref. [25].

Density functional theory (DFT) was performed for different approximant structural models that represent $i$-Al-Pd-Re. Depending on the model considered, its order, and its composition, a pseudogap $[32,35,43]$ or, in some cases, a narrow band gap was identified close to $E_{F}[32,43]$. Here, we demonstrate the occurrence of $\mathrm{AL}$ in $\mathrm{pg} i$-Al-Pd-Re that explains its unusual electronic structure and transport properties in comparison to sg $i$-Al-Pd-Re using hard x-ray photoemission spectroscopy (HAXPES). The large inelastic mean free path of electrons in HAXPES makes it immune to the possible surface effects, and this is particularly advantageous for studying the nature of the electron states near the Fermi level $E_{F}$ in quasicrystals $[38,39,44]$.

The valence band spectra. A comparison of the valence band (VB) spectra of sg and pg $i$-Al-Pd-Re in Fig. 1(a) shows differences that are quite unusual between two different forms of the same material. An interesting observation is the appearance of additional electron states at and around $E_{F}$ (shaded yellow) in pg, as also shown in an expanded scale in Fig. 1(b) for spectra recorded with a smaller step size and better signal-to-noise ratio. These extra states are shown by the difference spectrum (shaded green), and fitting by a Gaussian function gives its maximum at $0.26 \mathrm{eV}$ and FWHM of $0.6 \mathrm{eV}$. The states at $E_{F}\left[n\left(E_{F}\right)\right]$, highlighted by a circle, are enhanced by a factor of $\mathcal{I}=1.7 \mathrm{in}$ pg compared to sg. This is surprising because it apparently contradicts the resistivity behavior: $\rho$ of $\mathrm{pg}(\approx 22 \mathrm{~m} \Omega \mathrm{cm}$ at $4 \mathrm{~K})$ is substantially larger than that of $\mathrm{sg}(2.6 \mathrm{~m} \Omega \mathrm{cm})$ [Fig. 1(c)], which would imply suppression of states in pg [45]. The other notable dissimilarities in Fig. 1(a) are related to the peak around $4.5 \mathrm{eV}$ : it has larger intensity and is shifted by about $0.2 \mathrm{eV}$ towards lower binding energy (BE) in pg $i$-Al-Pd-Re. Moreover, suppression of states centered around $1.5 \mathrm{eV}$ (shaded pink) is observed.

The disparities in the shape of the VB are intriguing because neither their structure nor the strength of electronelectron correlation would differ between different forms of the same material. However, what could, indeed, differ is the degree of disorder. The monocrystalline sg specimen is expected to attain a thermodynamically stable ordered structure with equilibrium composition since it is melt grown by slow cooling. It exhibits a high degree of registry and minimal random phason strain $[24,25]$. On the other hand, rapid solidification and spark plasma sintering under high pressure used for preparation of pg could partially freeze the random phason strain that often involves site disorder [46]. In fact, phason strain has been reported in ingots of pg $i$-Al-Pd-Re [47]. Furthermore, the composition of pg $\left(\mathrm{Al}_{70.5} \mathrm{Pd}_{21} \mathrm{Re}_{8.5}\right)$ is somewhat different from $\mathrm{sg}\left(\mathrm{Al}_{73.5} \mathrm{Pd}_{17.1} \mathrm{Re}_{9.4}\right)$ with $3 \%$ $(0.9 \%)$ less $\mathrm{Al}(\mathrm{Re})$ and $3.9 \%$ excess Pd. This difference is corroborated by the relative intensities of the $\mathrm{Pd} 3 d$ and $\mathrm{Re}$ $4 d$ core-level peaks recorded by HAXPES (Fig. S1 in the Supplemental Material (SM) [48]). The deviation from the equilibrium sg stoichiometry along with the phason strain could give rise to site disorder in $\mathrm{pg}$.

Density functional theory. The DFT calculations [49-51] for $\mathrm{sg}$ and $\mathrm{pg} i$-Al-Pd-Re were performed using $2 / 1 \mathrm{ra}-$ tional approximants comprising overlapping pseudo-Mackay clusters (pMCs) and Bergman clusters according to the model by Katz-Gratias-Boudard (KGB) [52].

However, in the case of conflict in the overlapping region, the building principle of pMC is applied. We refer to this as the KGB+pMC model (see Discussion I (Methods) in the SM [48]). This model has been successful in obtaining the experimentally observed nonmagnetic ground state of Al-PdMn [53]. In the case of the sg, the $2 / 1 \mathrm{KGB}+\mathrm{pMC}$ model comprises $400 \mathrm{Al}, 92 \mathrm{Pd}$, and $52 \mathrm{Re}$ atoms in the unit cell (Al400-Pd92-Re52, i.e., $\mathrm{Al}_{73.5} \mathrm{Pd}_{16.9} \operatorname{Re}_{9.5}$, which is similar to the actual composition: $\left.\mathrm{Al}_{73.5} \mathrm{Pd}_{17.1} \operatorname{Re}_{9.4}\right)$. The pg structure is derived from sg in the same model by replacing 12- and 4-atom orbits of $\mathrm{Al}$, as well as a 4-atom orbit of $\mathrm{Re}$ by $\mathrm{Pd}$ atoms in sg, to arrive at a composition of Al384-Pd112-Re48, i.e., $\mathrm{Al}_{70.5} \mathrm{Pd}_{20.6} \mathrm{Re}_{8.8}$, which is very similar to the actual $\mathrm{pg}$ composition $\left(\mathrm{Al}_{70.5} \mathrm{Pd}_{21} \mathrm{Re}_{8.5}\right)$. Since the level of ordering is similar in both, the extra states at and around $E_{F}$ in pg [shaded yellow in Fig. 2(a)] in the total electronic density of states (DOS) is a consequence of their compositional difference. The increase in the DOS at $E_{F}$ is quantified by $\mathcal{I}=1.24$. The partial DOS (PDOS) in Figs. 2(b)-2(d) and S2 shows that $\mathcal{I} \geqslant 1$ in all cases but is most pronounced for $\mathrm{Re}$ and $\mathrm{Pd}$ PDOS; for example, $\operatorname{Re} 5 d, \operatorname{Pd} 4 d$, and $\operatorname{Pd} 5 p$ have $\mathcal{I}=1.44$, 1.25 , and 1.69 , respectively. In order to find the distribution of the atoms, we have calculated the radial distribution functions $g(r)$. In Figs. 3(a) and 3(b), the shells of $g(r)$ for the Pd-Re atom pair $g_{\mathrm{Pd}-\mathrm{Re}}(r)$ are well separated, showing similar levels of ordering. An interesting observation is that the nearest distance is substantially smaller in pg $(2.835 \AA)$ compared to sg 


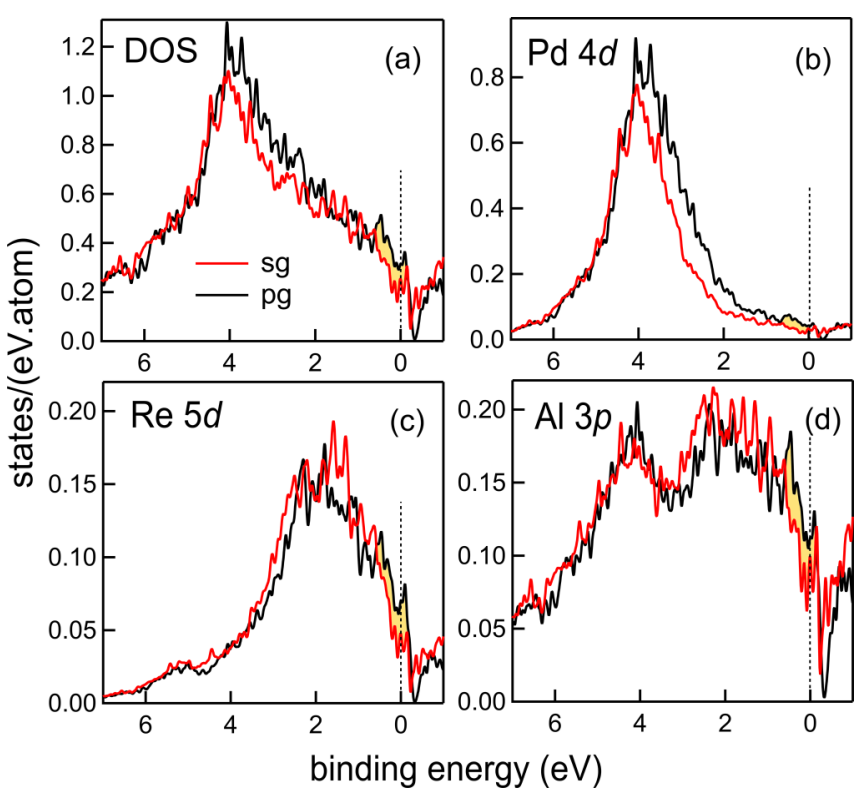

FIG. 2. (a) The total electronic density of states (DOS) and (b) Pd $4 d$, (c) Re $5 d$, and (d) Al $3 p$ partial DOS of sg and pg $i$-Al-PdRe (both are 2/1 KGB+pMC approximant models with 544 atoms per unit cell; see text). The extra states at and around $E_{F}$ in $\mathrm{pg}$ compared to sg $i$-Al-Pd-Re are shaded yellow.

(3.095 ̊). Larger $\mathcal{I}$ for the Re and Pd states and their smaller separation indicate enhanced hybridization of the $\mathrm{Pd}$ and $\mathrm{Re}$ states in pg that causes the occurrence of the extra states. The VBs calculated from the PDOS show reasonable agreement
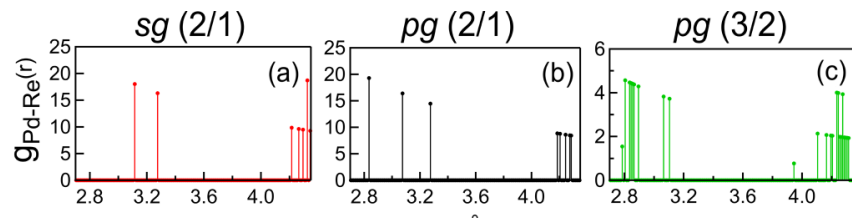

$r(\AA)$

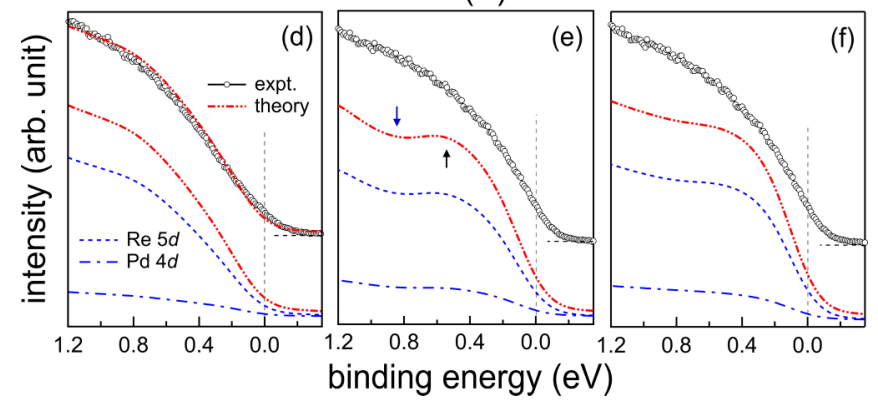

FIG. 3. The Pd-Re partial radial distribution functions $g_{\mathrm{Pd}-\mathrm{Re}}(r)$ are shown for (a) sg, (b) pg $i$-Al-Pd-Re (both are $2 / 1 \mathrm{KGB}+\mathrm{pMC}$ approximant models), and (c) pg with disorder (a 3/2 KGB approximant model); note that $g_{\mathrm{Pd}-\mathrm{Re}}(r)=0$ for $r<2.7 \AA$ for (a)-(c). The calculated VBs along with $\operatorname{Re} 5 d$ and $\mathrm{Pd} 4 d$ contributions are compared with the photoemission spectra in the near- $E_{F}$ region in (d)-(f) for each of the models, respectively. The spectra are staggered with the zero level shown by dashed horizontal lines on the right vertical axis. The calculated spectra are shifted by 0.16 and $0.09 \mathrm{eV}$ towards higher BE for sg and pg $i$-Al-Pd-Re, respectively. with experiment, and these extra states in pg are evident (see Discussion II in the SM and Fig. S3 [48]). Although shifted by $0.6 \mathrm{eV}$ in the calculations, the peak at $4.5 \mathrm{eV}$ in Fig. 1(a) can be assigned to the Pd $4 d$ states. It is more intense for $\mathrm{pg}$ because of the larger Pd content and is shifted towards lower BE by $0.18 \mathrm{eV}$ compared to sg (Fig. S3(c) [48]), in good agreement with experiment $(0.2 \mathrm{eV})$.

Effect of disorder on the $V B$. The shape of the calculated $\mathrm{VB}$ in the near- $E_{F}$ region [red dash-double-dotted curve in Fig. 3(d), also shown overlaid on the experimental VB (black open circles)] for sg exhibits excellent agreement with photoemission. This shows that the $2 / 1 \mathrm{KGB}+\mathrm{pMC}$ model describes it well. In the case of pg, the calculated VB shows a hump (at $0.4 \mathrm{eV}$, black arrow) and a dip [at $0.9 \mathrm{eV}$, blue arrow in Fig. 3(e)]. This hump-dip feature is observed for both $\operatorname{Re} d$ and $\mathrm{Pd} d$ states at the same energies, reconfirming the role of Re-Pd hybridization mentioned earlier. In contrast to the nice agreement in sg [Fig. 3(d)], the hump-dip feature in pg is not observed in the photoemission VB, which has a broad rounded shape [Fig. 3(e)].

It is well known that disorder leads to a smearing of the photoemission spectra with broadening of the features [54-57]. We probe whether the cause of the above disagreement might be related to disorder in pg by considering a $3 / 2$ approximant, albeit in the KGB model, where the absence of the building principle of the pMC clusters induces disorder, as explained below. In the KGB model, the positions of the atomic sites are obtained by a six-dimensional (6D) projection of triacontahedral acceptance domains of the $6 \mathrm{D}$ lattice. After relaxation of the interatomic forces, the largest deviation of the position of the atoms from their projected $6 \mathrm{D}$ positions is observed at atoms around the Re atoms in the centers of the pMC clusters. Thus, the KGB model exhibits an effective positional disorder compared to the $\mathrm{KGB}+\mathrm{pMC}$ model. Moreover, chemical or site disorder is also present in the KGB model since in the overlapping region of the pMC and Bergman clusters (see Fig. 18 of Ref. [53]), the chemical identity of the atoms is not well defined. The conflict arises because in the building principle of the $\mathrm{pMC}$ cluster, $\mathrm{Al}$ atoms occupy the inner shell with Re atoms at its center. In contrast, the building principle of the Bergman cluster requires a $\mathrm{Pd}$ atom at the $\mathrm{Al}$ position. Unfortunately, the structure of $i$-Al-Pd-Re has not been solved experimentally to date. The disorder of the KGB model is shown by $g_{\mathrm{Pd}-\mathrm{Re}}(r)$ in Fig. 3(c) when compared to that of KGB+pMC in Fig. 3(b): The first nearest-neighbor shell in $\mathrm{KGB}+\mathrm{pMC}$ comprises 12 Re atoms at $2.835 \AA$, whereas there are seven different closelying shells in KGB with distances varying from 2.785 to $2.895 \AA$, resulting in a substantially wider distribution. This is also observed around $4.2 \AA$. Such a wider distribution in the near-neighbor region is also observed in other $g(r)$ such as $g_{\mathrm{Re}-\mathrm{Re}}(r), g_{\mathrm{Pd}-\mathrm{Pd}}(r)$, and also, to some extent, $g_{\mathrm{Al}-\mathrm{Pd} / \mathrm{Re} / \mathrm{Al}}(r)$ (Fig. S4). This is the signature of effective disorder in the KGB model, in particular for Re and Pd. Site disorder is also present in Pd-Re that is created by substitution of some of the nearest $\mathrm{Al}$ neighbors of Re in the center of the $\mathrm{pMC}$ by $\mathrm{Pd}$ atoms. While in the ideal $\mathrm{KGB}+\mathrm{pMC}$ model there are no Pd-Re distances smaller than $2.9 \AA$, in models of pg such distances exist (Fig. 3). Finally, it may be noted that the 


\section{$\mathrm{T}(\mathrm{K})$}
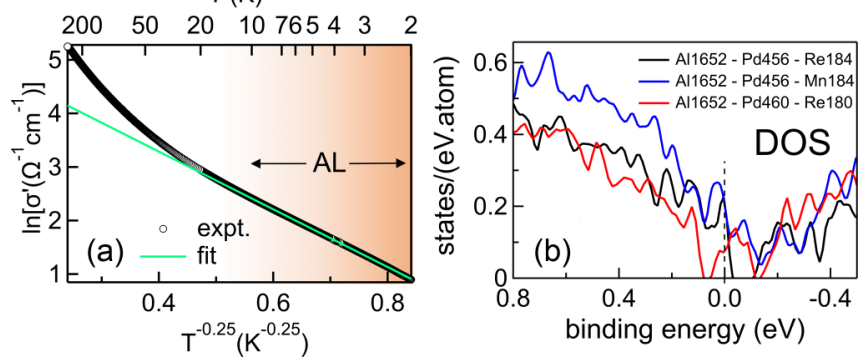

FIG. 4. (a) $\ln \sigma^{\prime}(T)$ vs $T^{-0.25}$ overlaid with a linear fit in the $2-10 \mathrm{~K}$ temperature range, indicating Mott variable range hopping in pg $i$-Al-Pd-Re. (b) The total DOS of 3/2 approximants of $i$-Al-Pd-Re and $i$-Al-Pd-Mn using the KGB+pMC model.

composition of the $3 / 2 \mathrm{KGB}$ model is Al1612-Pd472-Re208, i.e., $\mathrm{Al}_{70.33} \mathrm{Pd}_{20.59} \mathrm{Re}_{9.08}$, which is close to the actual composition of pg $i$-Al-Pd-Re.

For the KGB model in Fig. 3(f), the hump-dip feature observed for the KGB+pMC model is substantially reduced in the calculated VB. Also, the measure of the extra states at $E_{F}, \mathcal{I}=1.63$ for KGB, agrees better with experiment (1.7), in contrast to $\mathcal{I}=1.43$ for KGB + pMC (see Figs. S5 and S6 for DOS and the calculated VB over a wide range, respectively). Thus, the calculated VB for the KGB model with effective disorder is in better agreement with photoemission compared to the ordered $\mathrm{KGB}+$ pMC model. However, in Fig. 3(f), a close inspection reveals that the agreement is not perfect: the broad rounded shape of the photoemission VB is only partly reproduced by the KGB model. This indicates that in reality pg $i$-Al-Pd-Re might have stronger disorder; for example, random phason flips of atoms are not considered in our model.

Arguments for Anderson localization. The observation of extra electron states at $E_{F}$ in pg $i$-Al-Pd-Re in spite of its larger resistivity compared to sg $i$-Al-Pd-Re (Figs. 1 and 2) shows that these states should be localized. DFT furthermore shows that these states are broadened due to disorder (Fig. 3). Localized electron states in the presence of disorder indicate the possibility of AL. In fact, AL in three-dimensional quasicrystals with site disorder is supported by the tight-binding theory $[22,23]$. In order to confirm $\mathrm{AL}$ in our pg specimen, we noted that the Mott-type VRH behavior of conductivity $\sigma$ was suggested for AL states, where $\sigma(T)=\left[\sigma(0)+\sigma_{0} e^{\left[-\left(T_{0} / T\right)^{0.25}\right]}\right][58]$. Here, $\sigma(0)$ is the conductivity at $T=0, \sigma_{0}$ is a multiplicative factor, and $T_{0}$ is the activation temperature. The quality of the fit of $\sigma(T)$ for pg $i$-Al-Pd-Re is excellent: $\ln \left(\sigma^{\prime}\right)$ shows a straight line variation as a function of $T^{-0.25}$ up to $10 \mathrm{~K}$, confirming the occurrence of AL [Fig. 4(a)], where $\sigma^{\prime}=[\sigma-\sigma(0)]$. We find $\sigma(0)=40.56 \Omega^{-1} \mathrm{~cm}^{-1}$, in agreement with direct measurement [59], with $\rho(4.2 \mathrm{~K}) / \rho(300 \mathrm{~K})$ for our pg specimen being 5.2. Considering $T_{0}=839 \pm 3 \mathrm{~K}$ obtained from the fitting and $n\left(E_{F}\right)=0.315$ state $/ \mathrm{eV}$ atom from the DOS [Fig. 2(a)], we estimate the localization length $\xi=23 \AA$ using the relation $\xi^{3}=18 /\left[k_{B} T_{0} N\left(E_{F}\right)\right][4,60]$. Here, we establish Mott VRH in pg $i$-Al-Pd-Re up to $10 \mathrm{~K}$, whereas earlier reports are for a limited temperature range of up to $0.6 \mathrm{~K} \mathrm{[61]} \mathrm{and} 1.6 \mathrm{~K}$ [62]. Moreover, in this work, HAXPES and $\sigma$ measurements for the same ingot enable a direct comparison. We find that above $10 \mathrm{~K}, \sigma^{\prime}$ deviates [Fig. 4(a)], and the temperature range up to $280 \mathrm{~K}$ could be fitted using an expression almost linear in $T$, i.e., $\sigma=\sigma(0)+\sigma_{0} T^{n}, n=0.93$ (Fig. S7), as expected for a quasicrystal due to weak localization $[63,64]$.

In what follows, based on our data, we show why other possible explanations are not justifiable. Larger $\rho$ in pg $i$-AlPd-Re suggested [30] to be due to ES VRH can be examined from photoemission. A consequence of ES is that the shape of the VB near $E_{F}$, i.e., the spectral function $S(E)$, is proportional to $E^{2}$ [65], where $E$ is the binding energy. So we performed a least-squares fitting of the experimental spectrum of pg $i$-Al-Pd-Re in the near- $E_{F}$ region with $S(E) \propto E^{2}$ (Fig. S8) by varying all the relevant parameters. However, large systematic deviations are observed, and so the fitting fails. Moreover, for ES hopping the conductivity given by $\sigma(0)+\sigma_{0} e^{\left[-\left(T_{0} / T\right)^{0.5}\right]}[60,65]$ produces a worse fit than the Mott VRH (Fig. S9). Thus, the ES VRH can be ruled out for pg $i$-Al-Pd-Re based on both photoemission and transport measurements. An explanation based on the rigid-band model [33] is also not valid given the differences between the shapes of sg and pg VB [Figs. 1(a), 1(b), and 2]. Another suggestion that oxide formation at the grain boundaries enhances the resistivity of pg [35] does not hold because our core-level spectra do not show evidence of any such oxide formation (Fig. S10). Intrinsic grain boundary scattering also cannot explain such a large increase in $\rho$ of pg since the grain size is large $(10-30 \mu \mathrm{m})$.

Turning to the question of pseudogap in $i$-Al-Pd-Re, in Discussion III of the SM [48] and Fig. S11, we establish its existence in sg $i$-Al-Pd-Re. It is also shown that an inverted Lorentzian function describes its shape well by comparing with the near $E_{F}$ region of the calculated VB. A correlation between an increase of $\rho$ and the depth of the pseudogap $C_{L}$ for different $i$ quasicrystals, such as Al-Pd-Mn, Al-Cu-Fe, and $\mathrm{Zn}-\mathrm{Mg}$-Dy (none of these exhibit AL), has been shown [66]. sg $i$-Al-Pd-Re follows this general trend with $C_{L}=0.84$ (Fig. S11) and $\rho(4 K)=2-3 \mathrm{~m} \Omega \mathrm{cm}$ [25]. In contrast, the pseudogap is partially filled up by the extra states in pg. The conductivity of sg exhibits a $T^{1 / 2}$ power law behavior in the low-temperature range [25], i.e., $\sigma(T)=\sigma(0)+\sigma_{0} T^{1 / 2}$. The $\sigma(0)$ turns out to be $370 \Omega^{-1} \mathrm{~cm}^{-1}$, and this value is an order of magnitude larger than in pg.

From our DFT calculation, a pseudogap is observed above $E_{F}$ at $-0.23 \mathrm{eV}$ in sg $i$-Al-Pd-Re [Fig. 2(a)]. In the case of $\mathrm{pg}$, it also appears above $E_{F}$ at $-0.33(-0.38) \mathrm{eV}$ for the $2 / 1(3 / 2)$ approximant. It is clearly deeper in the KGB+pMC model, almost reaching zero, compared to the KGB model that has disorder [Figs. 2(a) and S5(a)]. Thus, a deeper pseudogap seems to occur in an ordered structural model. In order to probe this further, we consider an ideal $3 / 2$ $\mathrm{KGB}+\mathrm{pMC}$ model with composition Al1652-Pd456-Re184 (i.e., $\mathrm{Al}_{72.08} \mathrm{Pd}_{19.89} \mathrm{Re}_{8.03}$ ). A band gap with a width of $0.12 \mathrm{eV}$ just above $E_{F}$ is observed, which is a striking result for a unit cell comprising 2292 metal atoms [black curve in Fig. 4(b); also see Fig. S12(a)]. To explain the formation of the band gap, dimerization of the Re-Al-Pd-Al-Pd-Al-Re linear chain of covalent bonds, which connects the centers 
of pMC clusters occupied by Re, was proposed [32]. The present calculations show that even a small variation in composition, e.g., replacement of one four-atom orbit of Re by Pd (Al1652-Pd460-Re180), results in the appearance of states at $E_{F}$ flanked by narrow gaps at 0.07 and $-0.13 \mathrm{eV}$ [red curve in Fig. 4(b)]. This shows that the band gap in $i$-Al-Pd-Re depends sensitively on the atom positions and composition. The states in the gap created by such substitutional defects were shown to be localized in nature $[9,67]$. AL is favored at a band edge that would appear close to a gap [2], and DFT shows that this condition is satisfied in $i$-Al-Pd-Re because of the existence of a band gap [Fig. 4(b)]. Furthermore, the existence of Al-transition-metal linear chains in the $\mathrm{KGB}+\mathrm{pMC}$ model of $i$-Al-Pd-Re indicates that AL could be supported here with relatively less disorder, as indicated by early theoretical works $[68,69]$. Interestingly, the $3 / 2 \mathrm{KGB}+\mathrm{pMC}$ models do not have any Re-Pd nearest neighbors, do not show the extra states that are observed experimentally in pg, and largely resemble the sg experimental spectrum. The absence of Re-Pd nearest neighbors also explains why the pseudogap in sg is well formed, but due to compositional difference, a band gap is not observed experimentally.

Last, but not the least, to show that the existence of a band gap is unique for $i$-Al-Pd-Re, we performed a calculation for homologous $i$-Al-Pd-Mn (Al1652-Pd456-Mn184) by replacing Re with $\mathrm{Mn}$, with exactly the same structure and parameters as $i$-Al-Pd-Re (Al1652-Pd456-Re184). No band gap is observed for $i$-Al-Pd-Mn; rather, a pseudogap centered around $-0.15 \mathrm{eV}$ is observed [blue curve in Fig. 4(b)].

In conclusion, we observed a larger density of states at and around $E_{F}$ in pg $i$-Al-Pd-Re in comparison to sg $i$-Al$\mathrm{Pd}-\mathrm{Re}$ from photoemission, but the resistivity is one order of magnitude larger in the former. These two apparently contradictory experimental observations indicate that these states in pg $i$-Al-Pd-Re are localized. The conductivity measurement showed that the nature of localization is of the Mott VRH type, demonstrating the occurrence of Anderson localization. DFT showed that the extra states in pg arise from enhanced Re $5 d$ $\operatorname{Pd} 4 d$ hybridization and is related to the difference in their stoichiometries. But good agreement of the shape of the near- $E_{F}$ valence band spectrum of pg with DFT of the $3 / 2 \mathrm{KGB}$ approximant model (that has larger effective disorder) shows that these states are influenced by Pd-Re disorder [Fig. 3(f)]. An explanation from DFT for the possible origin of AL in Al-Pd-Re, in contrast to Al-Pd-Mn, comes from the existence of the semiconducting band gap in the former for an ordered $3 / 2 \mathrm{KGB}+\mathrm{pMC}$ model. In contrast, sg $i$-Al-Pd-Re exhibits a pseudogap at $E_{F}$, the depth of which is correlated with its conductivity as in other icosahedral quasicrystals. Its valence band agrees well with DFT for an ordered KGB + pMC model, indicating insignificant role of disorder. Thus, our work sheds light on the electronic structure of $i$-Al-Pd-Re, whose electronic properties have been under discussion for almost three decades. We hope that it will stimulate further theoretical research in disordered quasiperiodic systems, for example, the possible relation between the multifractal nature of the critical wave functions of quasiperiodic systems [70,71] and short-range fluctuations of the AL states [72]; the possible role of large spin-orbit coupling in $5 d$ metals such as Re could also be probed [73]. On the experimental front, the nice agreement between the photoemission VB and DFT for sg $i$-Al-Pd-Re indicates that the KGB $+\mathrm{pMC}$ model would serve as a good starting point for solving its structure.

Acknowledgments. The HAXPES experiments were carried out at the P09 beamline in PETRA III of DESY, a member of the Helmholtz-Gemeinschaft Deutscher Forschungszentren. Financial support by the DST, government of India, within the framework of India@ DESY collaboration is gratefully acknowledged. I. Tudosa is thanked for help with the growth of sg $i$-Al-Pd-Re measured in this study. M.K. thanks the Slovak Grant Agency VEGA (Grant No. 2/0082/17, 2/0144/21) and APVV (Grant No. 19-0369) for support. Part of the calculations was performed at the Computing Center of the Slovak Academy of Sciences using the supercomputing infrastructure acquired in Projects No. ITMS 26230120002 and No. 26210120002. A. Chakrabarti is thanked for useful discussions. J. Nayak, M. Maniraj, and S. Singh are thanked for help in some initial measurements. We are grateful to J. Delahaye, K. Horn, C. Schlueter, W. Drube, and C. Narayana for support and encouragement.
[1] P. W. Anderson, Phys. Rev. 109, 1492 (1958).

[2] P. A. Lee and T. V. Ramakrishnan, Rev. Mod. Phys. 57, 287 (1985).

[3] T. Siegrist, P. Jost, H. Volker, M. Woda, P. Merkelbach, C. Schlockermann, and M. Wuttig, Nat. Mater. 10, 202 (2011).

[4] T. Ying, Y. Gu, X. Chen, X. Wang, S. Jin, L. Zhao, W. Zhang, and X. Chen, Sci. Adv. 2, e1501283 (2016).

[5] C. Gómez-Navarro, P. J. D. Pablo, J. Gómez-Herrero, B. Biel, F. J. Garcia-Vidal, A. Rubio, and F. Flores, Nat. Mater. 4, 534 (2005).

[6] J. Liao, Y. Ou, X. Feng, S. Yang, C. Lin, W. Yang, K. Wu, K. He, X. Ma, Q.-K. Xue, and Y. Li, Phys. Rev. Lett. 114, 216601 (2015).

[7] G. Hertel, D. J. Bishop, E. G. Spencer, J. M. Rowell, and R. C. Dynes, Phys. Rev. Lett. 50, 743 (1983).
[8] S. von Molnar, A. Briggs, J. Flouquet, and G. Remenyi, Phys. Rev. Lett. 51, 706 (1983).

[9] Y. Zhang, R. Nelson, K.-M. Tam, W. Ku, U. Yu, N. S. Vidhyadhiraja, H. Terletska, J. Moreno, M. Jarrell, and T. Berlijn, Phys. Rev. B 98, 174204 (2018).

[10] D. Shechtman, I. Blech, D. Gratias, and J. W. Cahn, Phys. Rev. Lett. 53, 1951 (1984).

[11] A. P. Tsai, J. Q. Guo, E. Abe, H. Takakura, and T. J. Sato, Nature (London) 408, 537 (2000).

[12] A. I. Goldman and R. F. Kelton, Rev. Mod. Phys. 65, 213 (1993).

[13] A. I. Goldman, T. Kong, A. Kreyssig, A. Jesche, M. Ramazanoglu, K. W. Dennis, S. L. Bud'ko, and P. C. Canfield, Nat. Mater. 12, 714 (2013).

[14] K. J. Franke, H. R. Sharma, W. Theis, P. Gille, P. Ebert, and K. H. Rieder, Phys. Rev. Lett. 89, 156104 (2002). 
[15] V. K. Singh, M. Mihalkovic, M. Krajčí, S. Sarkar, P. Sadhukhan, M. Maniraj, A. Rai, K. Pussi, D. L. Schlagel, T. A. Lograsso, A. K. Shukla, and S. R. Barman, Phys. Rev. Res. 2, 013023 (2020).

[16] D. V. Talapin, E. V. Shevchenko, M. I. Bodnarchuk, X. Ye, J. Chen, and C. B. Murray, Nature (London) 461, 964 (2009).

[17] S. Förster, K. Meinel, R. Hammer, M. Trautmann, and W. Widdra, Nature (London) 502, 215 (2013).

[18] C. Xiao, N. Fujita, K. Miyasaka, Y. Sakamoto, and O. Terasaki, Nature (London) 487, 349 (2012).

[19] R. Chen, C.-Z. Chen, J.-H. Gao, B. Zhou, and D.-H. Xu, Phys. Rev. Lett. 124, 036803 (2020).

[20] D. Varjas, A. Lau, K. Pöyhönen, A. R. Akhmerov, D. I. Pikulin, and I. C. Fulga, Phys. Rev. Lett. 123, 196401 (2019).

[21] J. D. Cain, A. Azizi, M. Conrad, S. M. Griffin, and A. Zettl, Proc. Natl. Acad. Sci. USA 117, 26135 (2020).

[22] Y. K. Vekilov, E. I. Isaev, and S. F. Arslanov, Phys. Rev. B 62, 14040 (2000).

[23] M. Kohmoto and D. Tobe, Phys. Rev. B 77, 134204 (2008).

[24] J. Guo, T. Sato, E. Abe, H. Takakura, and A. Tsai, Philos. Mag. Lett. 80, 495 (2000).

[25] I. R. Fisher, X. P. Xie, I. Tudosa, C. W. Gao, C. Song, P. C. Canfield, A. Kracher, K. Dennis, D. Abanoz, and M. J. Kramer, Philos. Mag. B 82, 1089 (2002).

[26] S. Takeuchi, H. Akiyama, N. Naito, T. Shibuya, T. Hashimoto, K. Edagawa, and K. Kimura, J. Non-Cryst. Solids 153-154, 353 (1993).

[27] I. Kanazawa, H. Kitahata, and Y. Sekiyama, Z. Kristallogr. 223, 813 (2008).

[28] R. Kondo, T. Hashimoto, K. Edagawa, S. Takeuchi, T. Takeuchi, and U. Mizutani, J. Phys. Soc. Jpn. 66, 1097 (1997).

[29] F. S. Pierce, S. J. Poon, and Q. Guo, Science 261, 737 (1993).

[30] O. Rapp and S. J. Poon, Phys. Rev. B 84, 174206 (2011).

[31] O. Rapp, V. Srinivas, and S. J. Poon, Phys. Rev. B 71, 012202 (2005).

[32] M. Krajčí and J. Hafner, Phys. Rev. B 75, 024116 (2007).

[33] O. Rapp, Phys. Rev. B 94, 024202 (2016).

[34] F. S. Pierce, Q. Guo, and S. J. Poon, Phys. Rev. Lett. 73, 2220 (1994).

[35] J. Dolinšek, P. J. McGuiness, M. Klanjšek, I. Smiljanić, A. Smontara, E. S. Zijlstra, S. K. Bose, I. R. Fisher, M. J. Kramer, and P. C. Canfield, Phys. Rev. B 74, 134201 (2006).

[36] S. J. Poon and O. Rapp, Phys. Rev. B 76, 216201 (2007).

[37] J. Dolinšek, P. J. McGuiness, M. Klanjšek, I. Smiljanić, A. Smontara, E. S. Zijlstra, S. K. Bose, I. R. Fisher, M. J. Kramer, and P. C. Canfield, Phys. Rev. B 76, 216202 (2007).

[38] M. Gierer, M. A. Van Hove, A. I. Goldman, Z. Shen, S.-L. Chang, C. J. Jenks, C.-M. Zhang, and P. A. Thiel, Phys. Rev. Lett. 78, 467 (1997).

[39] G. Neuhold, S. R. Barman, K. Horn, W. Theis, P. Ebert, and K. Urban, Phys. Rev. B 58, 734 (1998).

[40] T. Fujiwara and T. Yokokawa, Phys. Rev. Lett. 66, 333 (1991).

[41] J. Hafner and M. Krajčí, Phys. Rev. Lett. 68, 2321 (1992).

[42] Z. M. Stadnik, D. Purdie, M. Garnier, Y. Baer, A. P. Tsai, A. Inoue, K. Edagawa, and S. Takeuchi, Phys. Rev. Lett. 77, 1777 (1996).

[43] M. Krajčí and J. Hafner, Phys. Rev. B 59, 8347 (1999).

[44] J. Nayak, M. Maniraj, A. Rai, S. Singh, P. Rajput, A. Gloskovskii, J. Zegenhagen, D. L. Schlagel, T. A. Lograsso, K. Horn, and S. R. Barman, Phys. Rev. Lett. 109, 216403 (2012).
[45] U. Mizutani, J. Phys.: Condens. Matter 10, 4609 (1998).

[46] P. M. Horn, W. Malzfeldt, D. P. DiVincenzo, J. Toner, and R. Gambino, Phys. Rev. Lett. 57, 1444 (1986).

[47] C. Beeli, C. Soltmann, and S. Poon, Mater. Sci. Eng. A 294 296, 531 (2000).

[48] See Supplemental Material at http://link.aps.org/supplemental/ 10.1103/PhysRevB.103.L241106 for Figs. S1- S12, Table S1, Discussions I-III, and Refs. [74-83].

[49] G. Kresse and J. Furthmüller, Phys. Rev. B 54, 11169 (1996).

[50] G. Kresse and D. Joubert, Phys. Rev. B 59, 1758 (1999).

[51] J. P. Perdew, K. Burke, and M. Ernzerhof, Phys. Rev. Lett. 77, 3865 (1996).

[52] A. Katz and D. Gratias, J. Non-Cryst. Solids 153-154, 187 (1993).

[53] M. Krajčí and J. Hafner, Phys. Rev. B 78, 224207 (2008).

[54] R. J. Cole, N. J. Brooks, and P. Weightman, Phys. Rev. Lett. 78, 3777 (1997).

[55] S. R. Barman and D. D. Sarma, Phys. Rev. B 51, 4007 (1995).

[56] P. Sadhukhan, S. W. D’Souza, V. K. Singh, R. S. Dhaka, A. Gloskovskii, S. K. Dhar, P. Raychaudhuri, A. Chainani, A. Chakrabarti, and S. Roy Barman, Phys. Rev. B 99, 035102 (2019).

[57] H. C. Robarts, T. E. Millichamp, D. A. Lagos, J. Laverock, D. Billington, J. A. Duffy, D. O’Neill, S. R. Giblin, J. W. Taylor, G. Kontrym-Sznajd, M. Samsel-Czekała, H. Bei, S. Mu, G. D. Samolyuk, G. M. Stocks, and S. B. Dugdale, Phys. Rev. Lett. 124, 046402 (2020).

[58] N. F. Mott, Philos. Mag. 19, 835 (1969).

[59] M. Ahlgren, C. Gignoux, M. Rodmar, C. Berger, and O. Rapp, Phys. Rev. B 55, R11915 (1997).

[60] Q. Guo and S. J. Poon, Phys. Rev. B 54, 12793 (1996).

[61] J. Delahaye, J. P. Brison, and C. Berger, Phys. Rev. Lett. 81, 4204 (1998).

[62] R. Rosenbaum, S.-T. Lin, and T.-I. Su, J. Phys.: Condens. Matter 15, 4169 (2003).

[63] C. Janot, Phys. Rev. B 53, 181 (1996).

[64] J. S. Dugdale, The Electrical Properties of Disordered Metals (Cambridge University Press, Cambridge, 1995).

[65] A. L. Efros and B. I. Shklovskii, J. Phys. C 8, L49 (1975).

[66] S. Sarkar, P. Sadhukhan, V. K. Singh, A. Gloskovskii, K. Deguchi, N. Fujita, and S. R. Barman, Phys. Rev. Res. 3, 013151 (2021).

[67] M. Krajčí and J. Hafner, Phys. Rev. B 68, 165202 (2003).

[68] N. Mott and W. Twose, Adv. Phys. 10, 107 (1961).

[69] R. E. Borland, Proc. R. Soc. London, Ser. A 274, 529 (1963).

[70] T. Fujiwara, M. Kohmoto, and T. Tokihiro, Phys. Rev. B 40, 7413 (1989).

[71] N. Macé, A. Jagannathan, P. Kalugin, R. Mosseri, and F. Piéchon, Phys. Rev. B 96, 045138 (2017).

[72] M. Schreiber and H. Grussbach, Phys. Rev. Lett. 67, 607 (1991).

[73] A. A. Starikov, P. J. Kelly, A. Brataas, Y. Tserkovnyak, and G. E. W. Bauer, Phys. Rev. Lett. 105, 236601 (2010).

[74] J. Strempfer, S. Francoual, D. Reuther, D. K. Shukla, A. Skaugen, H. Schulte-Schrepping, T. Kracht, and H. Franz, J. Synchrotron Radiat. 20, 541 (2013).

[75] M. Krajčí, M. Windisch, J. Hafner, G. Kresse, and M. Mihalkovič, Phys. Rev. B 51, 17355 (1995).

[76] M. Trzhaskovskaya and V. Yarzhemsky, At. Data Nucl. Data Tables 119, 99 (2018). 
[77] J. Nayak, M. Maniraj, A. Gloskovskii, M. Krajčí, S. Sebastian, I. R. Fisher, K. Horn, and S. R. Barman, Phys. Rev. B 91, 235116 (2015).

[78] S. Tougaard, Surf. Sci. 216, 343 (1989).

[79] H. A. E. Hagelin, J. F. Weaver, G. B. Hoflund, and G. N. Salaita, J. Electron Spectrosc. Relat. Phenom. 124, 1 (2002).

[80] J. E. Chae, J.-S. Kim, S.-Y. Nam, M. S. Kim, and J. Park, Appl. Micros. 50, 2 (2020).
[81] A. Östlin, L. Vitos, and L. Chioncel, Phys. Rev. B 98, 235135 (2018).

[82] A. Östlin, W. H. Appelt, I. Di Marco, W. Sun, M. Radonjić, M. Sekania, L. Vitos, O. Tjernberg, and L. Chioncel, Phys. Rev. B 93, 155152 (2016).

[83] W. Humphrey, A. Dalke, and K. Schulten, J. Mol. Graphics 14, 33 (1996). 